\title{
Optimal Tracing of Viscous Shocks in Solutions of Viscous Conservation Laws
}

\author{
Wen Shen and Mee Rea Park \\ Department of Mathematics, Penn State University, U.S.A. \\ e-mails: shen_w@math.psu.edu, park_m@math.psu.edu
}

May 25, 2006

\begin{abstract}
This paper contains a qualitative study of a scalar conservation law with viscosity:$$
u_{t}+f(u)_{x}=u_{x x} .
$$

We consider the problem of identifying the location of viscous shocks, thus obtaining an optimal finite dimensional description of solutions to the viscous conservation law. We introduce a nonlinear functional whose minimizers yield the viscous travelling profiles which "optimally fit" the given solution. We prove that, outside an initial time interval and away from times of shock interactions, our functional remains very small, i.e. the solution can be accurately represented by a finite number of viscous travelling waves.
\end{abstract}

Keywords: Optimal viscous shock tracing, viscous conservation laws, viscous travelling shocks, data compression, finite dimensional representation.

\section{Introduction}

Consider a scalar conservation law with viscosity

$$
u_{t}+f(u)_{x}=u_{x x} .
$$

We assume that the flux $f$ is smooth and genuinely nonlinear, so that $f^{\prime \prime}(u) \geq \kappa>0$ for every $u$. Our main interest here is: how to identify the emergence of viscous shocks in a solution, and how to optimally trace their locations and strengths.

More generally, one may ask the following question: Assume that a particular solution $u=u(t, x)$ has already been computed. If we are allowed only a finite number of parameters in order to describe its most relevant features, what is the best way to compress the information? In the literature, the problem of finite dimensional approximation of a dynamical system has been studied mainly by looking at $\omega$-limit sets $[T]$. Several results, valid for evolution equations of parabolic type, provide estimates on the dimension of an attractor. Of course, this yields a bound on the number of parameters needed to describe the evolution of the system asymptotically as $t \rightarrow+\infty$. 
In the present paper, the focus is different. Namely, we seek a finite dimensional description which is not only accurate in the asymptotic limit as $t \rightarrow+\infty$, but also in the "transient" regime. For solutions to a scalar, viscous conservation law, this transient behavior is actually the most interesting feature that can be observed. On the other hand, at least in the case of convex flux, the $\omega$-limit set is rather trivial. The asymptotic limit of any solution $t \mapsto u(t, \cdot)$ can be described in terms of the solution of a Riemann problem, i.e. either a single rarefaction or a viscous shock wave.

The problem of optimal location of viscous shock profiles was mentioned also in $[\mathrm{W}]$. In this connection, we introduce a scalar functional whose minimizers identify the strengths and locations of viscous shock profiles present in the solution. We also prove that, outside a set of times with finite measure, at all other times our functional has very small values. In other words, the description of the solution profile $u(t, \cdot)$ in terms of finitely many viscous shocks is accurate, for most times $t$. The exceptional set consists of an initial time interval and times at which shock interactions occur, see Figure 1.

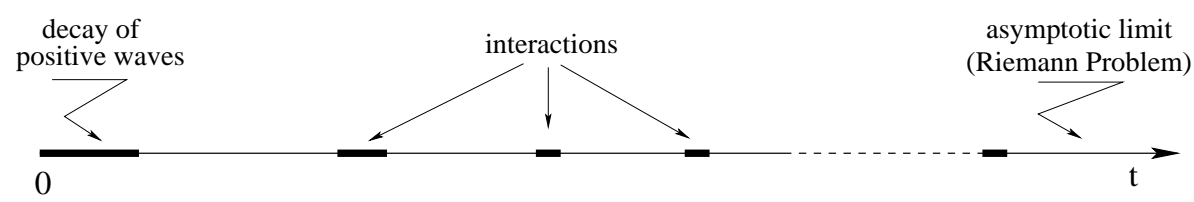

Figure 1: The exceptional set of times where the finite dimensional representation is not accurate.

\section{The main result}

We consider here the single conservation law with viscosity

$$
u_{t}+f(u)_{x}=u_{x x} .
$$

We fix $M>0$ and let $\mathcal{F}_{M}$ denote the set of all solutions to the Cauchy problem for (2.1) with initial data

$$
u(0, x)=\bar{u}(x)
$$

satisfying

$$
\text { Tot.Var. }\{\bar{u}\} \leq M, \quad\|\bar{u}\|_{\mathbf{L}^{\infty}} \leq M .
$$

We shall assume that the flux $f$ is $\mathcal{C}^{2}$ and strictly convex, so that $f^{\prime \prime}(u)>0$ for all $u \in \mathbb{R}$. In particular, this implies that there exist constants $\kappa, \kappa^{\prime}$,

$$
0<\kappa \leq f^{\prime \prime}(u) \leq \kappa^{\prime} \quad \text { for all } u \in[-M, M] .
$$

In essence, what we want to show is the following. Apart from a small set of times $J \subset[0, \infty[$, the profile $u(t, \cdot)$ of any solution of $(2.1)$ can be accurately described in terms of the "superposition" of finitely many travelling viscous shocks. Indeed, the assumption (2.4) of genuine non-linearity implies that all rarefaction waves will decay within an initial time interval. Moreover, in regions where the gradient $u_{x}$ is large and negative, viscous shock profiles will form. These can travel for a long time without much changing their shape, except when they interact with each other. The set $J$ of 
"exceptional times" where our description is not accurate will thus include an initial time interval, and also the intervals where wave interactions occur. Much of the following analysis aims at making rigorous the above claims.

For every $u^{-}>u^{+}$and $y \in \mathbb{R}$, let $\omega^{\left(u^{ \pm}, y\right)}$ be the unique viscous shock profile joining the states $u^{-}, u^{+}$, centered at $y$. This profile can be found as the unique solution to the O.D.E.

$$
\omega^{\prime}=f(\omega)-\sigma \omega-\left[f\left(u^{-}\right)-\sigma u^{-}\right], \quad \sigma=\frac{f\left(u^{-}\right)-f\left(u^{+}\right)}{u^{-}-u^{+}},
$$

satisfying the additional conditions

$$
\omega^{\prime \prime}(y)=0, \quad \omega(-\infty)=u^{-}, \quad \omega(+\infty)=u^{+} .
$$

Notice that the last two identities in (2.6) follow from (2.5) and the convexity of $f$. Given any solution $u \in \mathcal{F}_{M}$ of the conservation law, for each $t>0$ we introduce a description based on optimal location of shock profiles. Fix an integer $N \geq 1$ and let $\omega_{i}=\omega^{\left(u_{i}^{ \pm}, y_{i}\right)}$ be the $i$-th viscous shock profile we try to fit in. We consider the functional

$$
\begin{aligned}
\mathcal{J}\left(u(t), \omega_{1}, \ldots, \omega_{N}\right) \doteq \sum_{i=1}^{N} & \int_{\mathbb{R}}\left|u(t, x)-\omega_{i}(x)\right| \cdot\left|\omega_{i, x}(x)\right|^{2} d x \\
& +\int_{\mathbb{R}}\left|u_{x}(t, x)-\sum_{i=1}^{N} \omega_{i, x}(x)\right|^{2} d x
\end{aligned}
$$

Notice that the first integral measures the distance between $u$ and the travelling viscous shock $\omega_{i}$, multiplied by a weight function $\left|\omega_{i, x}\right|^{2}$ which is vanishingly small away from the center of the $i$-th shock. The second integral measures how well the derivative $u_{x}$ is approximated by derivatives of travelling shock profiles.

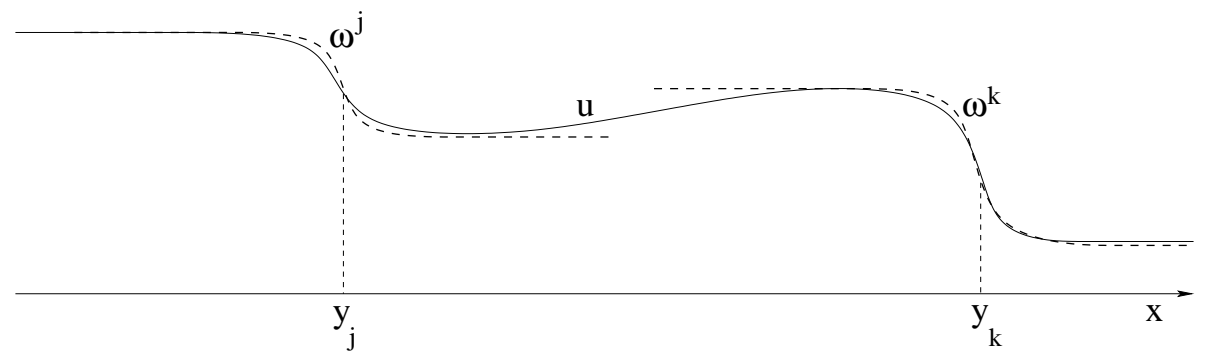

Figure 2: Fitting two viscous shocks $\omega_{j}, \omega_{k}$ in a solution.

If we fix a priori the complexity of our description, i.e. the integer $N$, how small can we render the integral $\mathcal{J}$ ? This problem can be formulated as

$$
\inf _{\omega_{1}, \ldots, \omega_{N}} \mathcal{J}\left(u(t), \omega_{1}, \ldots, \omega_{N}\right)
$$

where the infimum is taken over all $N$-tuples of travelling shock profiles $\omega_{i}=\omega^{\left(u_{i}^{ \pm}, y_{i}\right)}$, for some states $u_{i}^{-}>u_{i}^{+}$and $y_{i} \in \mathbb{R}$. Notice that if we choose $\omega_{i} \equiv 0$ for $i=1, \ldots, N$ (i.e., all travelling waves of zero amplitude), then the first integral in (2.7) vanishes because 
trivially $\omega_{i, x} \equiv 0$. However, in this case the second integral equals $\left\|u_{x}(t, \cdot)\right\|_{\mathbf{L}^{2}}^{2}$, which is of order Tot.Var. $(u)^{3}$ due to regularization, and can be large.

To estimate the quantity in (2.8), an intuitive argument goes as follows. Set $\delta=M / N$, where $M$ is given in (2.3). Since the total variation of $u(t)$ is bounded by $M$, there can be at most $N$ shock profiles of strength $\geq \delta$. Each one of these can be traced accurately. In addition, there may be an arbitrarily large number of smaller shocks, say of strengths $\sigma_{j}, j \geq 1$, with

$$
\sigma_{j} \leq \delta, \quad \sum_{j} \sigma_{j} \leq M .
$$

Each shock which is not traced produces an error in the second integral of (2.7) of the order

$$
\int\left|\omega_{j, x}(x)\right|^{2} d x=\mathcal{O}(1) \cdot \sigma_{j}^{3} .
$$

Because of (2.9) we thus expect that the minimum of $\mathcal{J}$ is approximately

$$
\mathcal{J}_{\text {min }} \approx \mathcal{O}(1) \cdot M \delta^{2}=\mathcal{O}(1) \cdot \frac{M^{3}}{N^{2}}
$$

The estimate (2.11) should indeed hold outside an initial time interval, where positive waves will decay, and away from interaction times. Our main results are as follows.

Theorem 1. Assume $f^{\prime \prime}(u) \geq \kappa>0$ for every $u \in \mathbb{R}$. Let $u \in \mathcal{F}_{M}$ be a solution of the viscous conservation law (2.1), and fix $N \geq 1$. Then, for every $t>0$, the minimization problem (2.8) has at least one solution.

Theorem 2. There exist constants $\alpha$ (uniformly valid for all $N \geq 1$ and $u \in \mathcal{F}_{M}$ ) and $\beta=\beta_{N, M}$ (depending only on $N$ and $M$ ) such that

$$
\mathcal{J}_{\min }(u(t)) \leq \alpha \cdot \frac{1}{N^{2}}
$$

for all $t \in\left[0, \infty\left[\backslash I^{u}\right.\right.$, for an exceptional set $I^{u}$ of times, with meas $\left(I^{u}\right) \leq \beta$.

The remainder of the paper contains a proof of the above two theorems. We remark that Theorem 1 states the existence of a minimizer for the scalar function $\mathcal{J}: \mathbb{R}^{3 N} \mapsto \mathbb{R}$. Since $\mathcal{J}$ is continuous and positive, the result would be trivial if $\mathcal{J}(y) \rightarrow \infty$ as $|y| \rightarrow \infty$. However, it is easily seen that this coercivity condition fails. The heart of the proof consists in showing that, if $\left\{X^{(m)}\right\}_{m \geq 1}$ is a minimizing sequence with $\left|X^{(m)}\right| \rightarrow \infty$, then a second minimizing sequence $\tilde{X}^{(m)}$ can be defined (in terms of $X^{(m)}$ ) whose elements remain uniformly bounded.

The proof of Theorem 2 involves a deeper argument. With a solution of the viscous equation (2.1) we associate a curve $\gamma$ moving in the plane. By results in [BB, BB1, BB2], the total area swept by this curve in its motion is a priori bounded in terms of a monotone decreasing area functional $Q(u)$. We then show that, at every time $t$ where the rate of decrease $\frac{d}{d t} Q(u(t))$ is sufficiently small, the inequality (2.12) holds. 
We remark that, in (2.8), the integer $N$ is fixed. Of course, one could let $N$ vary and look at the minimization problem

$$
\min _{N \geq 0} \inf _{\omega_{1}, \ldots, \omega_{N}}\left\{\epsilon N+\mathcal{J}\left(u(t), \omega_{1}, \ldots, \omega_{N}\right)\right\} .
$$

Here the first term penalizes the complexity of the description, adding a cost for each new viscous profile. The small constant $\epsilon>0$ acts as a threshold parameter. Small viscous shock waves, whose strength $\left\|\omega_{x}\right\|_{\mathbf{L}^{2}}^{2}$ is of order $<\epsilon$, will not be traced. From Theorem 1 it immediately follows that the problem (2.13) also admits a global minimizer. This can be interpreted as an "optimal description" of the solution profile $u(t, \cdot)$ as "superposition" of travelling viscous shocks.

\section{Proof of Theorem 1}

1. At any fixed time $t>0$, the solution $u(t, \cdot)$ of the viscous conservation law $(2.1)$ is a $C^{1}$ function with bounded total variation. We shall prove, more generally, that the functional $\mathcal{J}\left(u ; \omega_{1}, \ldots \omega_{N}\right)$ admits a global minimum for every $\mathcal{C}^{1}$ function $u: \mathbb{R} \mapsto \mathbb{R}$ with bounded variation.

2. Recall that $\omega_{k} \doteq \omega^{\left(u_{k}^{ \pm}, y_{k}\right)}$. Observing that the travelling wave profiles $\omega_{k}$ as well as their derivatives $\omega_{k, x}$ depend continuously on the scalar parameters $u_{k}^{-}, u_{k}^{+}, y_{k}$, $(k=1, \ldots, N)$, we have to prove that the continuous scalar function $\mathcal{J}(u ; \cdot): \mathbb{R}^{3 N} \mapsto \mathbb{R}$ admits a global minimum.

Since $\mathcal{J} \geq 0$, this function has a non-negative infimum $\mathcal{J}^{\text {min }}$. We can thus construct a minimizing sequence in $\mathbb{R}^{3 N}$, converging to $J^{\text {min }}$, say

$$
\left\{X^{(m)} \doteq\left(y_{1}^{(m)}, u_{1}^{(m)+}, u_{1}^{(m)-}, \ldots, y_{N}^{(m)}, u_{N}^{(m)+}, u_{N}^{(m)-}\right) ; m \geq 1\right\} .
$$

By possibly taking a subsequence, we can assume that each component of the vector $y_{m} \in \mathbb{R}^{3 N}$ either converges to a finite limit, or else diverges to $\pm \infty$.

3. If

$$
\sup _{m \geq 1}\left\{\left|y_{k}^{(m)}\right|+\left|u_{k}^{(m)+}\right|+\left|u_{k}^{(m)-}\right|\right\}<\infty
$$

for each $k=1, \ldots, N$, then the entire minimizing sequence $\left\{X^{(m)}\right\}_{m \geq 1}$ is bounded in $\mathbb{R}^{3 N}$. By our previous assumption, it converges to some limit

$$
\bar{X}=\left(\bar{y}_{1}, \bar{u}_{1}^{+}, \bar{u}_{1}^{-}, \ldots, \bar{y}_{N}, \bar{u}_{N}^{+}, \bar{u}_{N}^{-}\right) .
$$

By continuity, we thus have $\mathcal{J}(u ; \bar{X})=\mathcal{J}^{\text {min }}$, proving the existence of a minimizer.

4. In general, however, one cannot guarantee the minimizing sequence to be bounded, because the function $\mathcal{J}(u ; \cdot)$ is not coercive on $\mathbb{R}^{3 N}$. We shall thus adopt an alternative strategy. Assume that, for some index $j$,

$$
\lim _{m \rightarrow \infty}\left\{\left|y_{j}^{(m)}\right|+\left|u_{j}^{(m)+}\right|+\left|u_{j}^{(m)-}\right|\right\}=\infty .
$$


Consider the new sequence

$$
\tilde{X}^{(m)} \doteq\left(\tilde{y}_{1}^{(m)}, \tilde{u}_{1}^{(m)+}, \tilde{u}_{1}^{(m)-}, \ldots, \tilde{y}_{N}^{(m)}, \tilde{u}_{N}^{(m)+}, \tilde{u}_{N}^{(m)-}\right)
$$

obtained by setting the parameters of the $j$-th travelling profile to zero. More precisely, for every $m \geq 1$ we set

$$
\begin{aligned}
& \left(\tilde{y}_{i}^{(m)}, \tilde{u}_{i}^{(m)+}, \tilde{u}_{i}^{(m)-}\right)=\left(y_{i}^{(m)}, u_{i}^{(m)+}, u_{i}^{(m)-}\right) \quad \text { if } i \neq j, \\
& \left(\tilde{y}_{j}^{(m)}, \tilde{u}_{j}^{(m)+}, \tilde{u}_{j}^{(m)-}\right)=(0,0,0) .
\end{aligned}
$$

We claim that

$$
\limsup _{m \rightarrow \infty} \mathcal{J}\left(u ; \tilde{X}^{(m)}\right) \leq \lim _{m \rightarrow \infty} \mathcal{J}\left(u ; X^{(m)}\right) .
$$

If the original sequence had $k$ unbounded components, say for $j \in\left\{i_{1}, i_{2}, \ldots, i_{k}\right\} \subset$ $\{1,2, \ldots, N\}$, the above construction yields a new minimizing sequence having $k-1$ unbounded components. By induction, in a finite number of steps we obtain a minimizing sequence where all components are bounded. Hence, by step 3, a global minimizer exists.

5. It now remains to show that (3.2) holds. Equivalently, for every $\varepsilon>0$ we will prove that

$$
\limsup _{m \rightarrow \infty} \mathcal{J}\left(u ; \tilde{X}^{(m)}\right) \leq \lim _{m \rightarrow \infty} \mathcal{J}\left(u ; X^{(m)}\right)+\varepsilon .
$$

We shall consider different cases.

CASE 1: assume that, as $m \rightarrow \infty$,

$$
\left\|\omega_{j, x}^{(m)}\right\|_{\mathbf{L}^{2}} \rightarrow 0 .
$$

By the assumption $f^{\prime \prime}(u) \geq \kappa>0$, the strict convexity of the flux function implies

$$
\left\|\omega_{j, x}^{(m)}\right\|_{\mathbf{L}^{\infty}} \rightarrow 0 .
$$

In this case, observing that $\omega_{i, x}^{(m)} \leq 0$ because all viscous shock profiles are decreasing, we have the estimate

$$
\begin{aligned}
& \int_{-\infty}^{\infty}\left|u_{x}-\sum_{i=1}^{N} \omega_{i, x}^{(m)}\right|^{2} d x \\
& \geq \int_{-\infty}^{\infty}\left|u_{x}-\sum_{i \neq j} \omega_{i, x}^{(m)}\right|^{2} d x-2 \int_{-\infty}^{\infty}\left|u_{x}\right| \cdot\left|\omega_{j, x}^{(m)}\right| d x \\
& \geq \int_{-\infty}^{\infty}\left|u_{x}-\sum_{i \neq j} \omega_{i, x}^{(m)}\right|^{2} d x-2 \cdot\left\|u_{x}\right\|_{\mathbf{L}^{1}} \cdot\left\|\omega_{j, x}^{(m)}\right\|_{\mathbf{L}^{\infty}}
\end{aligned}
$$


Here we used the elementary inequality $(a-b-c)^{2} \geq(a-b)^{2}-2|a c|$, valid whenever $b$ and $c$ have the same sign. Therefore,

$$
\lim _{m \rightarrow \infty} \int_{-\infty}^{\infty}\left|u_{x}-\sum_{i=1}^{N} \omega_{i, x}^{(m)}\right|^{2} d x \geq \limsup _{m \rightarrow \infty} \int_{-\infty}^{\infty}\left|u_{x}-\sum_{i \neq j} \omega_{i, x}^{(m)}\right|^{2} d x,
$$

provided that (3.4) holds. Clearly, (3.6) implies (3.2). Notice that the condition (3.4) is certainly satisfied if $u^{(m)+}$ and $u^{(m)-}$ remain uniformly bounded and $\left|u^{(m)+}-u^{(m)-}\right| \rightarrow 0$.

CASE 2: assume that

$$
\liminf _{m \rightarrow \infty}\left\|\omega_{j, x}^{(m)}\right\|_{\mathbf{L}^{2}} \doteq \delta_{2}>0 .
$$

This breaks down into three different sub-cases.

CASE 2a: we have the limits $\left|y_{j}^{(m)}\right| \rightarrow \infty$ while $u_{j}^{(m)+} \rightarrow u_{j}^{+}, u_{j}^{(m)-} \rightarrow u_{j}^{-}$. To fix the ideas, assume $y_{j}^{(m)} \rightarrow+\infty$. Observe that in this case $\delta_{2}=\left\|\omega_{j, x}\right\|_{\mathbf{L}^{2}}$, where $\omega_{j}$ is a viscous shock profile connecting $u_{j}^{-}$with $u_{j}^{+}$. Given $\varepsilon>0$, choose $L$ so large that

$$
\left(\int_{L}^{\infty}\left|u_{x}\right|^{2} d x\right)^{1 / 2}<\frac{\varepsilon}{2 \delta_{2}}
$$

We then have the estimate

$$
\begin{gathered}
\int_{-\infty}^{\infty}\left|u_{x}-\sum_{i=1}^{N} \omega_{i, x}^{(m)}\right|^{2} d x \\
\geq \int_{-\infty}^{\infty}\left|u_{x}-\sum_{i \neq j} \omega_{i, x}^{(m)}\right|^{2} d x-2 \int_{-\infty}^{L}\left|u_{x}\right| \cdot\left|\omega_{j, x}^{(m)}\right| d x-2 \int_{L}^{\infty}\left|u_{x}\right| \cdot\left|\omega_{j, x}^{(m)}\right| d x \\
\geq \int_{-\infty}^{\infty}\left|u_{x}-\sum_{i \neq j} \omega_{i, x}^{(m)}\right|^{2} d x-2\left(\int_{-\infty}^{L}\left|u_{x}\right| d x\right) \cdot \sup _{x<L}\left|\omega_{j, x}^{(m)}(x)\right| \\
\quad-2\left(\int_{L}^{\infty}\left|u_{x}\right|^{2} d x\right)^{1 / 2} \cdot\left\|\omega_{j, x}^{(m)}\right\|_{\mathbf{L}^{2}} .
\end{gathered}
$$

Observing that

$$
\lim _{m \rightarrow \infty} \sup _{x<L}\left|\omega_{j, x}^{(m)}(x)\right|=0, \quad \quad \lim _{m \rightarrow \infty}\left\|\omega_{j, x}^{(m)}\right\|_{\mathbf{L}^{2}}=\delta_{2}
$$

from the above estimate we deduce

$$
\liminf _{m \rightarrow \infty} \int_{-\infty}^{\infty}\left|u_{x}-\sum_{i=1}^{N} \omega_{i, x}^{(m)}\right|^{2} d x \geq \liminf _{m \rightarrow \infty} \int_{-\infty}^{\infty}\left|u_{x}-\sum_{i \neq j} \omega_{i, x}^{(m)}\right|^{2} d x-2 \frac{\varepsilon}{2 \delta_{2}} \delta_{2} .
$$

This clearly implies (3.3). 
CASE 2b: assume that both sequences $u_{j}^{(m)+}$ and $u_{j}^{(m)-}$ diverge to $+\infty$. The case where they both tend to $-\infty$ is entirely similar. We then have

$$
\begin{aligned}
\liminf _{m \rightarrow \infty} & \int_{-\infty}^{\infty}\left|u-\omega_{j}^{(m)}\right| \cdot\left|\omega_{j, x}^{(m)}\right|^{2} d x \\
& \geq \lim _{m \rightarrow \infty}\left(\inf _{x \in \mathbb{R}}\left|u(x)-\omega_{j}^{(m)}(x)\right|\right) \cdot\left\|\omega_{j, x}^{(m)}\right\|_{\mathbf{L}^{2}}^{2}=\infty .
\end{aligned}
$$

Hence the original sequence was not minimizing. This contradiction shows that this case cannot happen.

CASE 2c: assume that the strength of the $j$-th travelling wave becomes arbitrarily large as $m \rightarrow \infty$, so that $u_{j}^{(m)-}-u_{j}^{(m)+} \rightarrow \infty$. In this case, it is easy to check that $\mathcal{J}\left(u ; X^{(m)}\right) \rightarrow \infty$. Indeed, let $K \doteq\|u\|_{\mathbf{L}^{\infty}}$. We then have

$$
\liminf _{m \rightarrow \infty} \int_{-\infty}^{\infty}\left|u-\omega_{j}^{(m)}\right| \cdot\left|\omega_{j, x}^{(m)}\right|^{2} d x \geq \liminf _{m \rightarrow \infty} \int\left|\omega_{j}^{(m)}(x)\right|>K+1\left|\omega_{j, x}^{(m)}\right|^{2} d x .
$$

Obviously, this integral diverges to infinity. Indeed, let's consider the case when

$$
\lim _{m \rightarrow \infty} u^{(m)-}=+\infty
$$

For the case when $\lim _{m \rightarrow \infty} u^{(m)+}=-\infty$ it is entirely similar. We have

$$
\begin{array}{r}
\liminf _{m \rightarrow \infty} \int\left|\omega_{j}^{(m)}(x)\right|>K+1 \\
\geq \liminf _{m \rightarrow \infty} \min _{\omega_{j}^{(m)}(x)>K+1}\left|\omega_{j, x}^{(m)}\right|^{2} d x \geq\left.\liminf _{m \rightarrow \infty} \int_{\omega_{j}^{(m)}(x)>K+1}\left|\cdot \int_{\omega_{j}^{(m)}(x)>K+1}\right| \omega_{j, x}^{(m)}\right|^{2} d x \\
\left|\omega_{j, x}^{(m)}\right| d x=\infty
\end{array}
$$

This proves that the original sequence was not minimizing. We again conclude that this case cannot happen. This completes the proof of Theorem 1.

\section{Proof of Theorem 2}

We shall rewrite the parabolic equation (2.1) using a different set of variables:

$$
v=f(u)-u_{x}, \quad \tau=t, \quad \eta=u .
$$

This change of variable was first introduced in $[\mathrm{BB}]$, and then used in later papers [BB1, BB2]. For each fixed time $t>0$, the solution of (2.1)-(2.2) is smooth. The map

$$
x \mapsto \gamma^{t}(x) \doteq(u(t, x), v(t, x))
$$

parameterizes a curve $\gamma^{t}$ in the $u-v$ plane. To see how this curve evolves in time, from (2.3) one obtains

$$
v_{t}+f^{\prime}(u) v_{x}=v_{x x} .
$$


On regions where $u_{x} \neq 0$ we can now use $(\tau, \eta)$ as independent variables, instead of $(t, x)$. From (4.1) and (4.3) we obtain

$$
\begin{gathered}
u_{x}=f(u)-v, \quad v_{\eta}=\frac{v_{x}}{u_{x}}, \quad v_{\eta \eta}=\frac{v_{x x}}{u_{x}^{2}}-\frac{v_{x}}{u_{x}^{3}} u_{x x}, \\
v_{\tau}=v_{t}-\frac{u_{t}}{u_{x}} v_{x}=\left(v_{x x}-f^{\prime}(u) v_{x}\right)-\frac{v_{x}}{u_{x}}\left(u_{x x}-f^{\prime}(u) u_{x}\right)=v_{x x}-\frac{u_{x x}}{u_{x}} v_{x} .
\end{gathered}
$$

Therefore

$$
v_{\tau}=\left(u_{x}\right)^{2} v_{\eta \eta}=(v-f(\eta))^{2} v_{\eta \eta} .
$$

In particular, the curve $\gamma=\gamma(\tau, \eta)=(\eta, v(\tau, \eta))$ evolves in the direction of the curvature and its total length is monotone decreasing in time. Another functional which is monotonically decreasing in time is the area functional

$$
Q(\gamma) \doteq \frac{1}{2} \iint_{\eta<\tilde{\eta}}\left|\gamma_{\eta}(\eta) \wedge \gamma_{\eta}(\tilde{\eta})\right| d \eta d \tilde{\eta},
$$

defined as the double integral of a wedge product. In terms of the original coordinates $u, x$, we have

$$
\begin{aligned}
Q(u)=\iint_{x<\tilde{x}} \mid u_{x}(\tilde{x}) \cdot\left[f^{\prime}(u(x)) \cdot u_{x}(x)-u_{x x}(x)\right] \\
-u_{x}(x) \cdot\left[f^{\prime}(u(\tilde{x})) \cdot u_{x}(\tilde{x})-u_{x x}(\tilde{x})\right] \mid d x d \tilde{x} .
\end{aligned}
$$

All these calculations (4.1-4.6) can be found in [BB, BB1]. As proved in [BB1], the decrease of the functional $Q$ controls the area swept by the curve $\gamma$ in its motion.

By parabolic regularization estimates, at time $t=1$ we now have

$$
Q(u(1)) \leq C_{1}
$$

for some constant $C_{1}$, uniformly valid for all solutions $u \in \mathcal{F}_{M}$. Therefore

$$
\begin{aligned}
\int_{1}^{\infty} \int(v-f(\eta))^{2}\left|v_{\eta \eta}\right| d \eta d \tau \leq \int_{1}^{\infty} \int\left|v_{\tau}(\tau, \eta)\right| d \eta d \tau \\
\leq \int_{1}^{\infty}-\left\{\frac{d}{d t} Q(u(t))\right\} d t \leq Q(u(1)) \leq C_{1} .
\end{aligned}
$$

As a consequence, for any given $\varepsilon>0$, there exists a set of times $I^{u} \subset[1, \infty[$ with

$$
\operatorname{meas}\left(I^{u}\right) \leq C_{1} / \varepsilon
$$

such that

$$
\int(v(t, \eta)-f(\eta))^{2} \cdot\left|v_{\eta \eta}(t, \eta)\right| d \eta \leq \varepsilon \quad \text { for all } t \geq 1, t \notin I^{u} .
$$

In addition, the assumption (2.4) of genuine nonlinearity yields the well known decay estimate $u_{x}(t, x) \leq(\kappa t)^{-1}$, hence

$$
-\infty<u_{x}(t, x) \leq \varepsilon \quad x \in \mathbb{R} .
$$



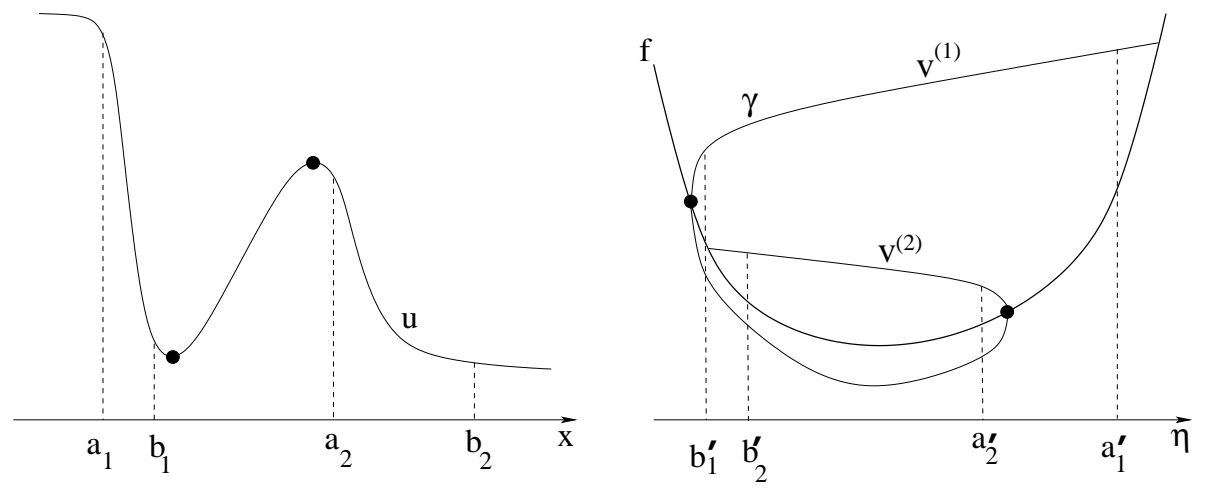

Figure 3: Example of a solution of viscous conservation law and the corresponding curve in $v-\eta$ plane.

for all $t \geq(\kappa \varepsilon)^{-1}$. To achieve a proof of Theorem 2, it now suffices to show that, at every time $t$ where (4.10)-(4.11) hold with some $\varepsilon>0$ sufficiently small, the profile of $u(t, \cdot)$ can be suitably approximated by a finite superposition of viscous shock profiles, and (2.12) holds.

As before, set $\delta \doteq M / N$. We can single out finitely many disjoint intervals $I_{k}=\left[a_{k}, b_{k}\right]$, $k=1, \ldots, \nu$, such that

$$
\begin{aligned}
\min _{x \in I_{k}} u_{x}(t, x) & \leq-7 \delta^{2} & & \text { for all } k, \\
u_{x}(t, x) \leq u_{x}\left(t, a_{k}\right)=u_{x}\left(t, b_{k}\right) & =-2 \delta^{3} & & \text { for all } x \in I_{k}, \\
u_{x}(t, x) & >-7 \delta^{2} & & \text { for all } x \notin I_{1} \cup \cdots \cup I_{\nu} .
\end{aligned}
$$

The images of these intervals through the mapping $x \mapsto \gamma(x)$ are graphs of functions $v=v^{(k)}(\eta)$, say with $\eta \in\left[b_{k}^{\prime}, a_{k}^{\prime}\right] \doteq\left[u\left(b_{k}\right), u\left(a_{k}\right)\right]$, see Figure 3 . For each $k$ we now choose a point $x_{k} \in\left[a_{k}, b_{k}\right]$ such that

$$
-m_{k} \doteq u_{x}\left(t, x_{k}\right)=\min _{a_{k} \leq x \leq b_{k}} u_{x}(t, x)
$$

and call $\gamma^{k}$ the segment in the $u-v$ plane with endpoints on the graph of the function $f$, tangent to the graph of the function $v^{(k)}$ at the point $c_{k} \doteq u\left(t, x_{k}\right)$, as in Figure 4 . Let $u_{k}^{+}<u_{k}^{-}$be the points where $\gamma^{k}$ intersects the graph of $f$, and call $\omega_{k}$ the unique viscous travelling wave profile satisfying

$$
\begin{array}{cl}
\omega(-\infty)=u_{k}^{-}, & \omega(\infty)=u_{k}^{+}, \\
\omega^{\prime}=f(\omega)-\sigma_{k} \omega-\left[f\left(u_{k}^{-}\right)-\sigma_{k} u_{k}^{-}\right], & \sigma_{k}=\frac{f\left(u_{k}^{-}\right)-f\left(u_{k}^{+}\right)}{u_{k}^{-}-u_{k}^{+}}=f^{\prime}\left(u\left(t, x_{k}\right)\right), \\
\omega\left(x_{k}\right)=u\left(t, x_{k}\right), & f^{\prime}\left(\omega\left(x_{k}\right)\right)=\sigma_{k} .
\end{array}
$$

It is important to notice that, by the previous construction, the image of the one-to-one map

$$
x \mapsto\left(\omega_{k}(x), f\left(\omega_{k}(x)\right)-\omega_{k, x}(x)\right)
$$




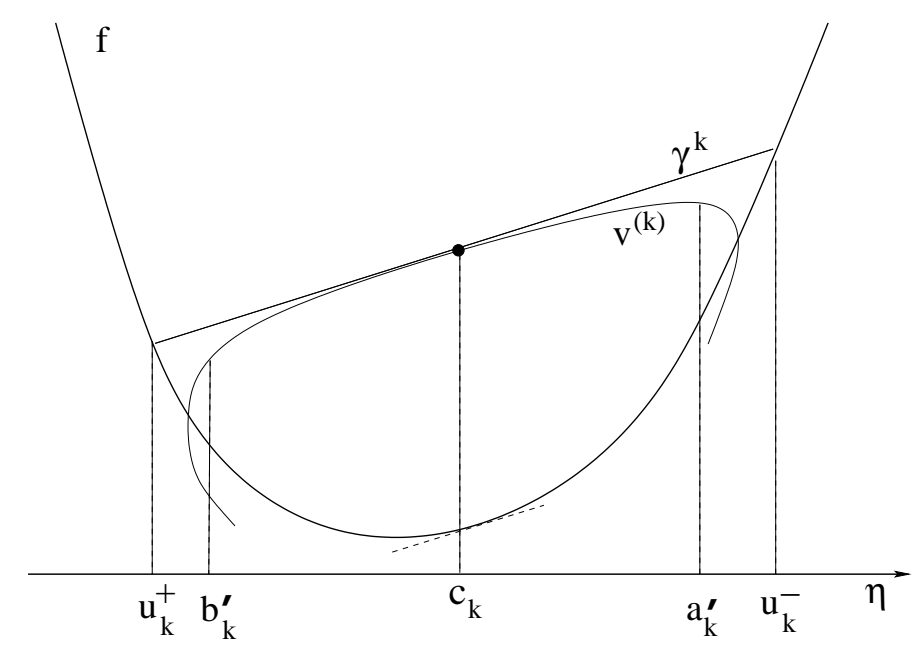

Figure 4: Fitting in a viscous shock $\omega_{k}$, illustrated in $v-\eta$ plane.

is precisely the segment $\gamma^{k}$. Moreover, the tangency condition and the maximality condition (4.14) imply that, at $x=x_{k}$,

$$
u_{x}\left(t, x_{k}\right)=\omega_{k, x}\left(x_{k}\right), \quad u_{x x}\left(t, x_{k}\right)=\omega_{k, x x}\left(x_{k}\right)=0 .
$$

Geometrically, this means that both $u(t, \cdot)$ and $\omega_{k}(\cdot)$ have an inflection point at $x=x_{k}$. We now recall that, by (4.10),

$$
\sum_{k} \int_{b_{k}^{\prime}}^{a_{k}^{\prime}}\left(v^{(k)}(\eta)-f(\eta)\right)^{2} \cdot\left|v_{\eta \eta}^{(k)}(t, \eta)\right| d \eta \leq \varepsilon .
$$

Restricted to the region where $u_{x} \leq-\delta^{3}$, the previous inequality implies the key estimate

$$
\sum_{k=1}^{\nu} \int_{\left\{v^{(k)}(\eta)-f(\eta) \geq \delta^{3}\right\}}\left|v_{\eta \eta}^{(k)}(t, \eta)\right| d \eta \leq \frac{\varepsilon}{\delta^{6}} .
$$

Since $\varepsilon>0$ can be chosen arbitrarily small, according to (4.17) every function $v^{(k)}$ is "almost affine", hence its graph is very well approximated by the tangent line $\gamma^{k}$. Reverting to the original variables $t, x$, this in turn implies that $u(t, \cdot)$ is closely approximated by the corresponding travelling profile $\omega_{k}$, on the appropriate interval $x \in\left[a_{k}, b_{k}\right]$.

Lemma 1. Assume that the flux function satisfies

$$
0<\kappa \leq f^{\prime \prime}(u) \leq \kappa^{\prime}, \quad \text { for all } u \in \mathbb{R} .
$$

Then for every $\varepsilon^{\prime}>0$ there exists $\varepsilon>0$ small enough so that (4.17) implies the following.

$$
\left\|u-\omega_{k}\right\|_{\mathbf{L}^{\infty}\left(\left[a_{k}, b_{k}\right]\right)} \leq \varepsilon^{\prime}, \quad\left\|u-\omega_{k}\right\|_{H^{1}\left(\left[a_{k}, b_{k}\right]\right)}^{2} \leq \varepsilon^{\prime}, \quad \text { for all } k .
$$

Moreover,

$$
u\left(t, a_{k}\right)-u\left(t, b_{k}\right) \geq \sqrt{\frac{m_{k}}{\kappa^{\prime}}}
$$




$$
\begin{aligned}
\sup _{x \notin\left[a_{k}, b_{k}\right]}\left|\omega_{k, x}(x)\right| & \leq 3 \delta^{3}, \\
\int_{\mathbb{R} \backslash\left[a_{k}, b_{k}\right]}\left|\omega_{k, x}(x)\right| d x & \leq \frac{6 \delta^{2}}{\sqrt{\kappa}} .
\end{aligned}
$$

Proof. By choosing $\varepsilon>0$ sufficiently small, we can assume that the $\mathcal{C}^{1}$ distance

$$
\left\|v^{(k)}-\gamma^{k}\right\|_{\mathcal{C}^{1}\left(\left[b_{k}^{\prime}, a_{k}^{\prime}\right]\right)}
$$

is as small as we like. By (4.12), when $x \in\left[a_{k}, b_{k}\right]$ we have $u_{x} \leq-2 \delta^{3}$. The map $x \mapsto u(x)$ is thus invertible on each interval $\left[a_{k}, b_{k}\right]$. The two norms in (4.19) can both be estimated in terms of the distance (4.23).

We now prove (4.20). Using (4.23) and recalling (4.12), by taking $\varepsilon>0$ sufficiently small we can assume that

$$
\begin{aligned}
& \gamma^{k}\left(a_{k}^{\prime}\right)-f\left(a_{k}^{\prime}\right) \leq v^{(k)}\left(a_{k}^{\prime}\right)-f\left(a_{k}^{\prime}\right)+\left\|v^{(k)}-\gamma^{k}\right\|_{\mathcal{C}^{0}} \leq 3 \delta^{3} \\
& \gamma^{k}\left(b_{k}^{\prime}\right)-f\left(b_{k}^{\prime}\right) \leq v^{(k)}\left(b_{k}^{\prime}\right)-f\left(b_{k}^{\prime}\right)+\left\|v^{(k)}-\gamma^{k}\right\|_{\mathcal{C}^{0}} \leq 3 \delta^{3}
\end{aligned}
$$

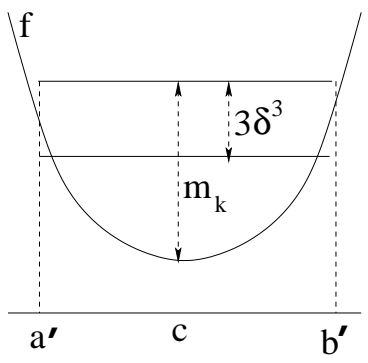

Figure 5: (a)

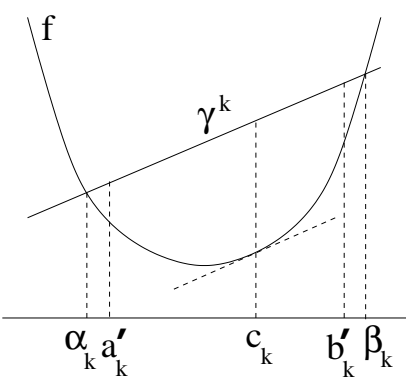

(b)

The inequality (4.20) now follows from a simple geometrical inequality (see Figure 5(a)). If $f^{\prime \prime}<\kappa^{\prime}$ and $\gamma$ is a linear function such that

$$
\gamma\left(a^{\prime}\right)-f\left(a^{\prime}\right) \leq 3 \delta^{3}, \quad \gamma\left(b^{\prime}\right)-f\left(b^{\prime}\right) \leq 3 \delta^{3}, \quad \gamma(c)-f(c)=m_{k},
$$

for some points $b^{\prime}<c<a^{\prime}$, then

$$
a^{\prime}-b^{\prime} \geq \sqrt{\frac{2\left(m_{k}-3 \delta^{3}\right)}{\kappa^{\prime}}} .
$$

Since we are assuming $\delta<1, m_{k} \geq 7 \delta^{2}>2\left(3 \delta^{3}\right)$, from the previous inequality we deduce

$$
a_{k}^{\prime}-b_{k}^{\prime} \geq \sqrt{\frac{m_{k}}{\kappa^{\prime}}}
$$

proving (4.20).

The inequality (4.21) follows from

$$
\sup _{x \notin\left[a_{k}, b_{k}\right]}\left|\omega_{k, x}(x)\right|=\max \left\{\left|\omega_{k, x}\left(a_{k}^{\prime}\right)\right|,\left|\omega_{k, x}\left(b_{k}^{\prime}\right)\right|\right\} \leq 2 \delta^{3}+\left\|v^{(k)}-\gamma^{k}\right\|_{\mathcal{C}^{1}\left(\left[b_{k}^{\prime}, a_{k}^{\prime}\right]\right)} \leq 3 \delta^{3} .
$$


To prove (4.22), call $\alpha_{k}, \beta_{k}$ the points where the line $\gamma^{k}$ intersects the graph of $f$, as in Figure 5(b). Then

$$
\int_{\mathbb{R} \backslash\left[a_{k}, b_{k}\right]}\left|\omega_{k, x}(x)\right| d x=\left(\alpha_{k}-a_{k}^{\prime}\right)+\left(b_{k}^{\prime}-\beta_{k}\right) .
$$

Consider the function $g(u) \doteq \gamma^{k}(u)-f(u)$. Clearly, we have

$$
g\left(c_{k}\right)=\max _{u} g(u) \geq 7 \delta^{2},
$$

and $c_{k}$ is the midpoint of the interval $\left[\beta_{k}, \alpha_{k}\right]$. Assuming $f^{\prime \prime} \geq \kappa$, then

$$
a_{k}^{\prime}-c_{k} \geq \frac{1}{2} \sqrt{\frac{4\left(m_{k}-3 \delta^{3}\right)}{\kappa}} .
$$

Recalling that

$$
g\left(a_{k}^{\prime}\right) \leq 3 \delta^{3}, \quad g\left(b_{k}^{\prime}\right) \leq 3 \delta^{3}, \quad g^{\prime \prime}=-f^{\prime \prime} \leq-\kappa,
$$

we conclude that

$$
-g^{\prime}\left(a_{k}^{\prime}\right)=-\int_{c_{k}}^{a_{k}^{\prime}} g^{\prime \prime}(u) d u \geq \kappa\left(a_{k}^{\prime}-c_{k}\right) \geq \sqrt{\kappa\left(m_{k}-3 \delta^{3}\right)} .
$$

Recalling that $m_{k} \geq 7 \delta^{2}$ and $\delta<1$, we obtain

$$
\alpha_{k}-a_{k}^{\prime} \leq \frac{3 \delta^{3}}{\sqrt{\kappa\left(m_{k}-3 \delta^{3}\right)}} \leq \frac{6 \delta^{3}}{\sqrt{8 \kappa \delta^{2}}} \leq \frac{3 \delta^{2}}{\sqrt{\kappa}} .
$$

The estimate for $b_{k}^{\prime}-\beta_{k}$ is totally similar. Together, these yield (4.22). The proof of the lemma is completed.

As approximations to $u(t, \cdot)$ we now choose the $N$ travelling profiles $\omega_{k}$ in the above list, corresponding to the $N$ largest values of $m_{k}$, say $m_{1} \geq m_{2} \geq \cdots \geq m_{N}$. Notice that (4.20) implies

$$
N \sqrt{\frac{m_{N}}{\kappa^{\prime}}} \leq M, \quad m_{N} \leq \kappa^{\prime}\left(\frac{M}{N}\right)^{2} .
$$

Hence

$$
\left|u_{x}(t, x)\right| \leq \kappa^{\prime}\left(\frac{M}{N}\right)^{2} \quad x \notin \bigcup_{k=1}^{N}\left[a_{k}, b_{k}\right] .
$$

Using the estimates (4.19)-(4.21) we now check that the functional $\mathcal{J}$ at (2.7) is small, as claimed by Theorem 2. The first half of the right hand side in (2.7) can be estimated as

$$
\begin{aligned}
& \sum_{k=1}^{N} \int_{\mathbb{R}}\left|u(t, x)-\omega_{k}(x)\right| \cdot\left|\omega_{k, x}(x)\right|^{2} d x \\
& \quad=\sum_{k=1}^{N}\left(\int_{x \in\left[a_{k}, b_{k}\right]}+\int_{x \notin\left[a_{k}, b_{k}\right]}\right)\left|u(t, x)-\omega_{k}(x)\right| \cdot\left|\omega_{k, x}(x)\right|^{2} d x
\end{aligned}
$$




$$
\begin{aligned}
\leq & \sum_{k}\left\|u-\omega_{k}\right\|_{\mathbf{L}^{\infty}\left(\left[a_{k}, b_{k}\right]\right)} \cdot\left\|\omega_{k, x}\right\|_{\mathbf{L}^{2}(\mathbb{R})}^{2} \\
& +\sum_{k}\left(\|u\|_{\mathbf{L}^{\infty}(\mathbb{R})}+\left\|\omega_{k}\right\|_{\mathbf{L}^{\infty}(\mathbb{R})}\right) \sup _{x \notin\left[a_{k}, b_{k}\right]}\left|\omega_{k, x}(x)\right| \cdot \int_{\mathbb{R} \backslash\left[a_{k}, b_{k}\right]}\left|\omega_{k, x}(x)\right| d x \\
\leq & \mathcal{O}(1) \cdot N \varepsilon^{\prime}+N \cdot 2 M \cdot 3 \delta^{3} \frac{6 \delta^{2}}{\sqrt{\kappa}}=\mathcal{O}(1) \cdot \frac{1}{N^{4}} .
\end{aligned}
$$

For the second half of the right hand side of (2.7), we can use the inequality $(a+b)^{2} \leq$ $2\left(a^{2}+b^{2}\right)$, valid for all real numbers of $a, b$, and we get

$$
\begin{aligned}
& \int_{\mathbb{R}}\left|u_{x}(t, x)-\sum_{k=1}^{N} \omega_{k, x}(x)\right|^{2} d x \\
& =\sum_{k} \int_{a_{k}}^{b_{k}}\left|u_{x}(t, x)-\sum_{k=1}^{N} \omega_{k, x}(x)\right|^{2} d x+\int_{\mathbb{R} \backslash \cup_{k}\left[a_{k}, b_{k}\right]}\left|u_{x}(t, x)-\sum_{k=1}^{N} \omega_{k, x}(x)\right|^{2} d x \\
& \leq \sum_{k} \int_{a_{k}}^{b_{k}}\left(\left|u_{x}(t, x)-\omega_{k, x}(x)\right|+\sum_{j \neq k}\left|\omega_{j, x}(x)\right|\right)^{2} d x \\
& \quad+\int_{\mathbb{R} \backslash \cup_{k}\left[a_{k}, b_{k}\right]}\left(\left|u_{x}(t, x)\right|+\sum_{k}\left|\omega_{k, x}(x)\right|\right)^{2} d x \\
& \leq \quad 2 \sum_{k}\left\{\int_{a_{k}}^{b_{k}}\left|u_{x}(t, x)-\omega_{k, x}(x)\right|^{2} d x+\int_{a_{k}}^{b_{k}}\left(\sum_{j \neq k}\left|\omega_{j, x}(x)\right|\right)^{2} d x\right\} \\
& \quad+2 \int_{\mathbb{R} \backslash \cup_{k}\left[a_{k}, b_{k}\right]}\left[\left|u_{x}(t, x)\right|^{2}+\left(\sum_{k}\left|\omega_{k, x}(x)\right|\right)^{2}\right] d x \\
& \leq \quad I_{1}+I_{2}+I_{3}+I_{4},
\end{aligned}
$$

where

$$
\begin{aligned}
& I_{1}=2 \sum_{k}\left|u-\omega_{k}\right|_{H^{1}\left(\left[a_{k}, b_{k}\right]\right)}, \\
& I_{2}=2 \sum_{j} \int_{\mathbb{R} \backslash\left[a_{j}, b_{j}\right]}\left|\omega_{j, x}(x)\right|^{2} d x \leq 2 \sum_{j}\left\{\sup _{x \notin\left[a_{j}, b_{j}\right]}\left|\omega_{j, x}(x)\right| \cdot \int_{\mathbb{R} \backslash\left[a_{j}, b_{j}\right]}\left|\omega_{j, x}(x)\right| d x\right\}, \\
& I_{3}=2\left\{\sup _{x \notin \cup\left[a_{k}, b_{k}\right]}\left|u_{x}(t, x)\right|\right\} \cdot \int_{\mathbb{R}}\left|u_{x}(t, x)\right| d x, \\
& I_{4}=2 \sum_{k}\left\{\sup _{x \notin\left[a_{k}, b_{k}\right]}\left|\omega_{k, x}(x)\right|\right\} \cdot \int_{\mathbb{R} \backslash \cup_{k}\left[a_{k}, b_{k}\right]} \sum_{k}\left|\omega_{k, x}(x)\right| d x .
\end{aligned}
$$

Using the estimates in Lemma 1 and (4.24), we get

$$
\begin{aligned}
& I_{1} \leq 2 N \varepsilon^{\prime}, \\
& I_{2} \leq 2 N 3 \delta^{3} \frac{6 \delta^{2}}{\sqrt{\kappa}},
\end{aligned}
$$




$$
\begin{aligned}
I_{3} & \leq 2 \kappa^{\prime} \frac{M^{2}}{N^{2}} M \\
I_{4} & \leq 2 \cdot N 3 \delta^{3} \cdot N \frac{6 \delta^{2}}{\sqrt{\kappa}} .
\end{aligned}
$$

Note that $I_{1}$ measures how well the viscous shock profile matches the solution on the interval $\left[a_{k}, b_{k}\right]$, and this term is arbitrary small. $I_{2}$ measures the $H_{1}$ norm of the viscous shock waves outside the interval $\left[a_{k}, b_{k}\right]$, and it is of $\mathcal{O}(1) / N^{4}$. And $I_{3}$ is the sum of all the shock waves that are not represented. This is the largest term here, and is of $\mathcal{O}(1) / N^{2}$. Finally, $I_{4}$ is similar to $I_{2}$, and is of $\mathcal{O}(1) / N^{3}$. In summary, we have

$$
\int_{\mathbb{R}}\left|u_{x}(t, x)-\sum_{k=1}^{N} \omega_{k, x}(x)\right|^{2} d x \leq \mathcal{O}(1) \cdot \frac{1}{N^{2}} .
$$

Putting these two parts together, we get the desired result.

\section{Concluding remarks}

For solutions to the conservation law (2.1), the transient behavior is nontrivial and can last an arbitrary long time. This happens because we are considering solutions defined on the whole real line. On the other hand, if the equation is restricted to a bounded interval, say

$$
\left.u_{t}+f(u)_{x}=u_{x x} \quad x \in\right] a, b[,
$$

with boundary conditions

$$
u(a)=\alpha, \quad u(b)=\beta,
$$

then all solutions would converge at an exponential rate to a unique steady state $w(\cdot)$. Indeed, from basic theory of parabolic equations $[\mathrm{H}]$ it follows that there exists a unique function $w:[a, b] \mapsto \mathbb{R}$ which satisfies the two-point boundary value problem

$$
f(w)_{x}=w_{x x}, \quad w(a)=\alpha, \quad w(b)=\beta .
$$

Linearizing (5.1) around the steady state $w$, one obtains the existence of some $\delta>0$ such that, for every initial data $\bar{u} \in \mathbf{L}^{2}$ the corresponding solution of (5.1)-(5.2) satisfies

$$
\|u(t)-w\|_{\mathcal{C}^{k}([a, b])} \leq C e^{-\delta t} .
$$

Here one can choose a constant $C$ uniformly valid on bounded subsets of $\mathbf{L}^{2}$. After an initial time interval, the long term behavior of the solution is thus trivial.

In the case of a bounded domain, the corresponding equation (4.4) in the $(\eta, v)$ variables must be supplemented with the boundary conditions

$$
v_{\eta}(\alpha)=v_{\eta}(\beta)=0 \text {. }
$$

The unique steady state solution of (5.3) corresponds to a constant function:

$$
v(\eta) \equiv \kappa \quad \text { for all } \eta \in[\alpha, \beta] .
$$


Observing that

$$
b-a=\int_{\alpha}^{\beta} \frac{1}{u_{x}} d u=\int_{\alpha}^{\beta} \frac{1}{f(\eta)-v(\eta)} d \eta,
$$

one can uniquely determine the constant $\kappa$ from the relation

$$
b-a=\int_{\alpha}^{\beta} \frac{1}{f(\eta)-\kappa} d \eta .
$$

Acknowledgment: The authors are grateful to Professor Alberto Bressan at the same department for proposing the problem, and for many useful discussions. We also want to thank one of the referees for carefully reading through the paper and for various remarks.

\section{References}

[BB] S. Bianchini and A. Bressan, A case study in vanishing viscosity, Discrete and Continuous Dynamic Systems, 7:3 (2001), 449-476.

[BB1] S. Bianchini and A. Bressan, On a Lyapunov functional relating shortening curves and viscous conservation laws, Nonlinear Analysis, T.M.A. 51 (2002), 649-662.

[BB2] S. Bianchini and A. Bressan, Vanishing viscosity solutions of nonlinear hyperbolic systems, Annals of Mathematics 161 (2005), 223-342.

[B] A. Bressan, Hyperbolic Systems of Conservation Laws. The One Dimensional Cauchy Problem, Oxford University Press, 2000.

[H] D. Henry, Geometric Theory of Semilinear Parabolic Equations, Lecture Notes in Mathematics 840, Springer-Verlag, New York, 1981.

[S] D. Serre, Systems of Conservation Laws I, Cambridge University Press, 2000.

[Sm] J. Smoller, Shock Waves and Reaction-Diffusion Equations, Springer-Verlag, New York, 1983.

[T] R. Temam, Infinite Dimensional Dynamical Systems in Mathematics and Physics, Springer-Verlag, New York, 1988.

[W] G. Whitham, Linear and Nonlinear waves, Wiley-Interscience, 1974. 